\title{
Perpendicular Axis
}

National Cancer Institute

\section{Source}

National Cancer Institute. Perpendicular Axis. NCI Thesaurus. Code C54649.

A straight line through a body or figure that is at a right angle to a given line or plane. 